\title{
Geometric accuracy evaluation of a six-degree-of-freedom (6-DoF) couch with cone beam computed tomography (CBCT) using a phantom and correlation study of the position errors in pelvic tumor radiotherapy
}

\author{
Caofei $\mathrm{Fu}^{1}$, Changsheng $\mathrm{Ma}^{2}$, Dongping Shang ${ }^{2}$, Qingtao Qiu ${ }^{2}$, Huipeng Meng ${ }^{3}$, Jinghao Duan $^{2}$, Yong Yin $^{2}$ \\ ${ }^{1}$ Department of Radiotherapy, Xiang'an Hospital of Xiamen University, School of Medicine, Xiamen University, Xiamen, China; ${ }^{2}$ Department of \\ Radiation Oncology, Shandong Cancer Hospital and Institute, Shandong First Medical University and Shandong Academy of Medical Sciences, \\ Ji'nan, China; ${ }^{3}$ School of Precision Instrument and Opto-electronics Engineering, Tianjin University, Tianjin, China \\ Contributions: (I) Conception and design: J Duan; (II) Administrative support: J Duan; (III) Provision of study materials or patients: C Fu; (IV) \\ Collection and assembly of data: C Fu, J Duan; (V) Data analysis and interpretation: C Fu, J Duan; (VI) Manuscript writing: All authors; (VII) Final \\ approval of manuscript: All authors. \\ Correspondence to: Jinghao Duan. Department of Radiation Oncology, Shandong Cancer Hospital and Institute, Shandong First Medical University \\ and Shandong Academy of Medical Sciences, Ji’nan, China. Email: jinghaoduan@126.com.
}

Background: To assess the position accuracy of the six-degree-of-freedom (6-DoF) couch based on cone beam computed tomography (CBCT) and exploit the correlation of the six degrees errors.

Methods: CT scans of an anthropomorphic phantom and patients were obtained at $3 \mathrm{~mm}$ slice thicknesses using a Philips scanner at the head, first supine and prostrate positions. An Eclipse Treatment Planning System was used to create a treatment plan. Different levels of known position errors were introduced to simulate patient position status for the anthropomorphic phantom. CBCT datasets for each position were acquired and registered to original CT datasets to evaluate the accuracy of the 6-DoF couch and determine the setup errors of patients. The setup errors of 200 CBCT datasets from 14 patients with pelvic tumors were analyzed. The correlations between six degrees position errors were finally extracted.

Results: For the phantom study, the difference between known introduced errors and the setup errors were almost negligible. The deviation (mean \pm one standard deviation) in registration methods were $(0.01 \pm 0.02) \mathrm{cm},(0.04 \pm 0.075) \mathrm{cm},(0.02 \pm 0.004) \mathrm{cm},(0.01 \pm 0.04)^{\circ},(0.1 \pm 0.08)^{\circ},(0.03 \pm 0.05)^{\circ}$ and $(0.01 \pm$ $0.01) \mathrm{cm},(0.03 \pm 0.007) \mathrm{cm},(0.01 \pm 0.01) \mathrm{cm},(0.05 \pm 0.06)^{\circ},(0.08 \pm 0.08)^{\circ},(0.04 \pm 0.05)^{\circ}$ for supine and prone position, respectively. The deviation in positions were $(0.07 \pm 0.10) \mathrm{cm},(0.16 \pm 0.02) \mathrm{cm},(0.08 \pm 0.06) \mathrm{cm}$, $(0.54 \pm 0.46)^{\circ},(0.24 \pm 0.16)^{\circ},(0.09 \pm 0.09)^{\circ}$ and $(0.06 \pm 0.09) \mathrm{cm},(0.19 \pm 0.09) \mathrm{cm},(0.09 \pm 0.07) \mathrm{cm},(0.49 \pm 0.49)^{\circ}$, $(0.16 \pm 0.08)^{\circ},(0.1 \pm 0.13)^{\circ}$ for bone and soft tissue registration methods, respectively. For patient data, the setup errors were $(-0.07 \pm 0.22) \mathrm{cm},(0.14 \pm 0.35) \mathrm{cm},(-0.12 \pm 0.4) \mathrm{cm},(0.79 \pm 1.6)^{\circ},(0.41 \pm 0.71)^{\circ},(-0.03 \pm 0.8)^{\circ}$ for supine position and $(0.16 \pm 0.27) \mathrm{cm},(0.19 \pm 0.48) \mathrm{cm},(-0.05 \pm 0.34) \mathrm{cm},(1.1 \pm 1.49)^{\circ},(0.65 \pm 1.00)^{\circ},(-0.23 \pm 0.75)^{\circ}$ for prone position, respectively. There is a significant moderate correlation between the longitudinal and pitch directions and between the vertical and pitch directions when the patient is in the supine position.

Conclusions: The six-dimensional couch positioning verification system based on CBCT has high accuracy and can meet the requirements of precise radiotherapy for pelvic tumors. There is a certain correlation between translation direction and rotation direction.

Keywords: Positioning error; six-degree-of-freedom (6-DoF) couch; cone beam computed tomography (CBCT); correlation analysis

Submitted Mar 16, 2020. Accepted for publication Aug 21, 2020.

doi: $10.21037 /$ tcr-20-1528

View this article at: http://dx.doi.org/10.21037/tcr-20-1528 


\section{Introduction}

Radiotherapy has been one of the most important methods of tumor treatment since the development of radiation equipment and physics. As general radiotherapy methods, the 3D-CRT, IMRT and VMAT technologies need highly accurate patient setups. The posture fixation of patients during treatment becomes a significant procedure, which has a direct impact on the positioning accuracy. Schmidhalter's (1) study found that high setup accuracy provided target margin reduction, which provides better protection for normal tissue and results in an improvement in patient care.

Over the past few decades, image guidance radiotherapy (IGRT) has integrated imaging systems with linear accelerators and achieved high patient positioning accuracy. IGRT technology can help us to observe three-dimensional (3D) anatomy and soft-tissue contrast information (2), which enables us to correct setup errors.

In an early IGRT system, a four-degree-of-freedom (4-DoF) couch was employed, which could only correct the setup errors concerning the three translational axeslongitudinal (long), lateral (lat) and vertical (vert)and the couch rotation angle (rot) (3). In recent years, a new IGRT system combining the six-degree-of-freedom (6-DoF) couch has been introduced into the clinic to further improve patient setup accuracy (3-8). Compared with the 4-DoF couch, the 6-DoF couch has two additional degrees of freedom, including rotational axes pitch and roll (3). Theoretically, the 6-DoF couch united with kilovoltage cone beam computed tomography (kV-CBCT) can further reduce setup errors. This may lead to a potential situation that decreases the clinical target volume (CTV) to planning target volume (PTV) margins $(9,10)$.

In recent years, some studies have reported that the 6-DoF couch based on CBCT can significantly reduce errors in stereotactic radiosurgery (SRS) and stereotactic body radiotherapy (SBRT) $(5,6,11,12)$. Patient setup errors may have a serious dosimetric influence on the tumor target (13). However, few studies have studied the positioning errors in the pelvis and the correlation between the six degrees of freedom shifts. Chiesa et al. reported that there was no correlation between translational and rotational errors in prostate treatment (2). However, pelvic tumors are unstable as a result of translational and rotational motion. In addition, there are many other deficiencies in positioning sourced from the patient's weight, abdominal respiration, postprandial time, bladder filling degree, and subcutaneous fat thickness. Thus, many positioning errors and poor repeatability often occur in pelvic tumor radiotherapy. Therefore, it is important to ensure position errors for tumor radiotherapy within acceptable limits.

Thus, the accurate evaluation of the 6-DoF couch appears to be particularly important for patient radiotherapy positioning. In this study, we designed an experiment utilizing an anthropomorphic phantom to verify the accuracy of the patient positioning error for a 6-DoF couch based on CBCT. In addition, we analyzed the error data to search for the correlation between six-dimensional setup errors in pelvic tumor patients. This study may be helpful to improve the positioning accuracy and reduce the margin of CTV to PTV in current clinical practice.

\section{Methods}

\section{6-DoF robotic couch patient positioning system}

All experiments and treatments were performed at the Varian VitalBeam Linear Accelerator (Varian Medical Systems, Inc., Palo Alto, CA), which is equipped with a kilovoltage CBCT image-guidance system and a 6-DoF couch. The 6-DoF couch combines yaw, pitch and roll rotational motions with translational motions and achieves submillimeter and subdegree precision in high-precision radiotherapy patient positioning.

\section{The setup experiment of anthropomorphic phantoms}

An anthropomorphic phantom [Alderson Rando phantom (14,15), Alderson Research Laboratories, Inc.] was used to obtain the setup errors in this experiment, which have shapes and anatomical characteristics that are similar to real patients with pelvic tumors who were treated with IMRT. The Alderson Rando phantom supine and prone positions are shown in Figure 1. The phantom was initially set up on the CT couch by comparing the cross mark between the room laser lamp and phantom.

A pelvic CT scan of the anthropomorphic phantom was obtained by using a Philips scanner (Holland, CT Lightspeed 16) with an imaging protocol of $120 \mathrm{kV}$ tube voltage, $300 \mathrm{~mA}$ cube current, $3 \mathrm{~mm}$ thickness and an in-plane resolution of $0.97 \times 0.97$ at the head, supine and prostrate positions. The structures and treatment plan were created on the CT-scan images by using the Eclipse Treatment Planning System (Version 13.6, Varian Medical Systems, Palo Alto, CA) in the supine and prone positions, 

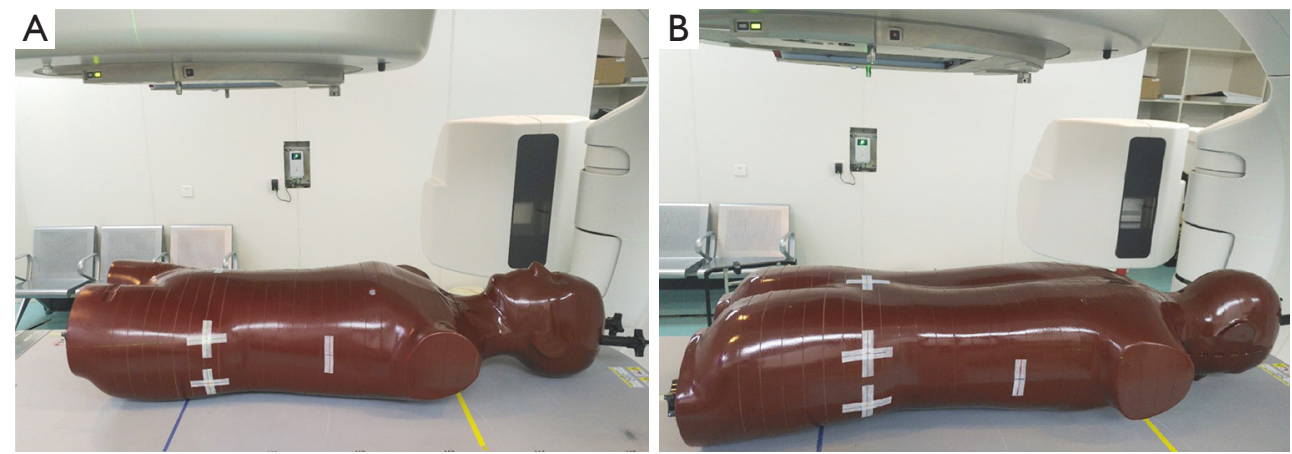

Figure 1 The anthropomorphic phantom: (A) supine position; (B) prone position.

Table 1 Error grading classification

\begin{tabular}{ll}
\hline Grades & Setup errors contents \\
\hline 0 & Error free \\
1 & Lateral shift $\pm 2 \mathrm{~cm}$ \\
2 & Lateral shift $\pm 2 \mathrm{~cm}$ and Longitudinal $\pm 3 \mathrm{~cm}$ \\
3 & Yaw $3^{\circ}$ (clockwise or counterclockwise directions) \\
4 & Pitch $2^{\circ}$ \\
5 & Yaw $3^{\circ}$ and Pitch $2^{\circ}$ \\
6 & Lateral shift $\pm 2 \mathrm{~cm}$ and Yaw $3^{\circ}$ and Pitch $2^{\circ}$ \\
\hline
\end{tabular}

respectively. After simulation of the CTV, the PTV was obtained by expanding the CTV according to the Radiation Therapy Oncology Group (RTOG) 4018 reports as follows: adding $5 \mathrm{~mm}$ in the mediolateral (ML) and posterior (P) directions and $8 \mathrm{~mm}$ in the superio-inferior (SI) and anterior (A) directions (9). Immediately, IMRT treatment plans were designed and scheduled. Then, the plans were transferred onto the Vitalbeam Linear Accelerator.

We introduced different levels of known position errors to simulate the patient positioning procedure. The phantom was intentionally shifted 2 and $3 \mathrm{~cm}$ in lateral and longitudinal directions, while the angular shifts of $2^{\circ}$ and $3^{\circ}$ were introduced in pitch and yaw directions. We defined the setup errors as six categories by the combination of directions as shown in Table 1, which hasn't any physical meaning and was used only for representation purposes. Then, CBCTs were performed and registered with the planning CT image to verify the shifts when the anthropomorphic phantom was placed on the linear accelerator table. The phantom was intentionally shifted in particular directions but remained constant in other directions. All of the above steps were repeated in supine and prone positions.

\section{The retrospective analysis of patients}

After the phantom experiment, we retrospectively analyzed the positioning errors of patients in the supine and prone positions. Two hundred verifications of 14 pelvic tumor patients who received radiotherapy on a Vitalbeam Linear Accelerator were randomly selected. All six couch axes were analyzed in this study. All the patients in our work were corrected by the 6-DoF couch with CBCT before the treatment delivery, in which the planning CT image was matched with the CBCT image acquired immediately using the default algorithm based on bone matching. The study was conducted in accordance with the Declaration of Helsinki (as revised in 2013). The ethics committee of Shandong Cancer Hospital and Institute approved this study (No.: 2019-371). Because this is not a treatmentbased study, our institutional review board waived the need for written informed consent from the participants.

The mean and the standard deviation of the shifts between the CBCT and the planning CT in translational and rotational directions were calculated, where the mean describes the systematic error and the standard deviation describes the random error. On the other hand, the Pearson correlation between two pairs of the patients' positioning error data in 6 directions (left-right direction: $\mathrm{X}$, inferiorsuperior direction: $\mathrm{Y}$, anterior-posterior direction: $\mathrm{Z}$, leftright rotation: roll, anterior-posterior rotation: pitch, counterclockwise or clockwise rotation: yaw) was performed to verify the findings of the phantom experiment.

Statistically, the correlation factor $r(-1 \leq r \leq 1)$ indicates the linear relationship between a dependent and an independent variable; 1 represents a perfect correlation, 0 represents no correlation, positive values represent a positive relationship 

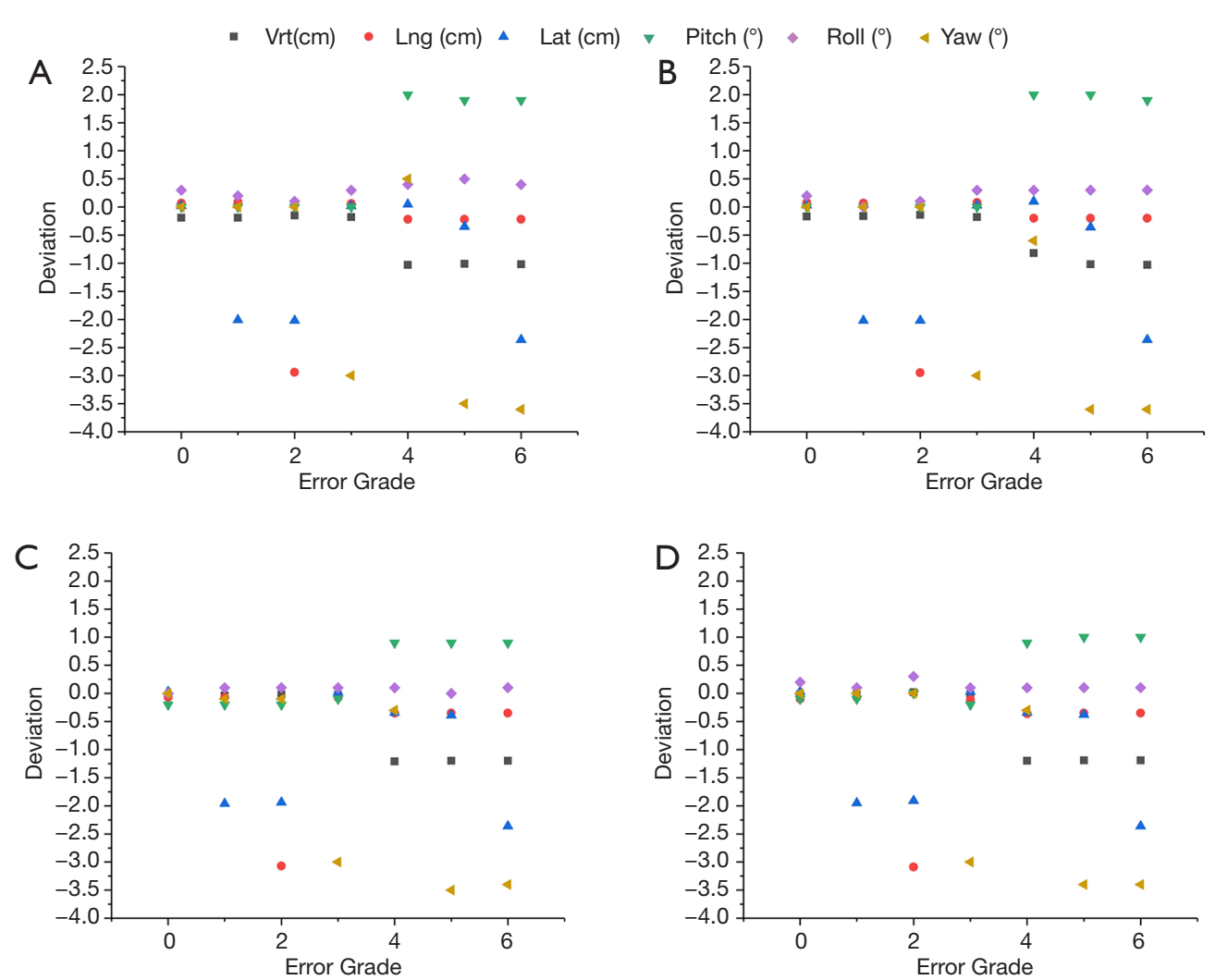

Figure 2 Scatter plot of absolute error in different positions and registration methods. (A) Scatter plot for bone registration in supine position; (B) scatter plot for soft tissue registration in supine position; (C) scatter plot for bone registration in prostrate position; (D) scatter plot for soft tissue registration in prostrate position.

(one increases as the other increases), and negative values represent a negative relationship (one increases as the other decreases). The ranges of $0<|r| \leq 0.4,0.4<|r| \leq 0.7$ and $0.7<|\mathrm{r}| \leq 1$ indicate weak, moderate and strong correlations between the above variables, respectively.

\section{Statistical analysis}

SPSS software, version 22.0 (SPSS, Chicago, IL, USA) was used to conduct statistical analysis. The data were consistent with the normal distribution, and statistical analysis was performed using the paired $t$-test or Wilcoxon rank sum test. It was considered statistically significant when the twotailed $\mathrm{P}$ value was $<0.05$.

\section{Results}

\section{Anthropomorphic phantom setup errors}

In this investigation, misalignments up to $2 \mathrm{~cm}$ in translational and $3^{\circ}$ rotational shifts were introduced to investigate the positioning errors, which was confirmed by the 6-DoF system registration of a CBCT to the planning CT. Considering the influence of position and registration method on positioning accuracy, we repeated the positioning procedure and recorded the errors, as shown in Figure 2. This indicates that the deviation of six introduced directions can be accurately detected after CBCT registration. The errors of translation direction may increase with the misalignment introduction of rotation direction, while the rotation errors would not be affected by the translation shifts. To study the error changes in different positions and different registration methods, we calculated the average and standard deviation of the absolute error value under different conditions, which is shown in Table 2. The average deviation in the translation direction between different registration methods does not exceed $0.04 \mathrm{~mm}$, regardless of the supine or prone position. The rotation direction is less than $0.1^{\circ}$. When bone registration or soft tissue matching is punctual, the average deviation of 
Table 2 The deviation in different position and registration methods

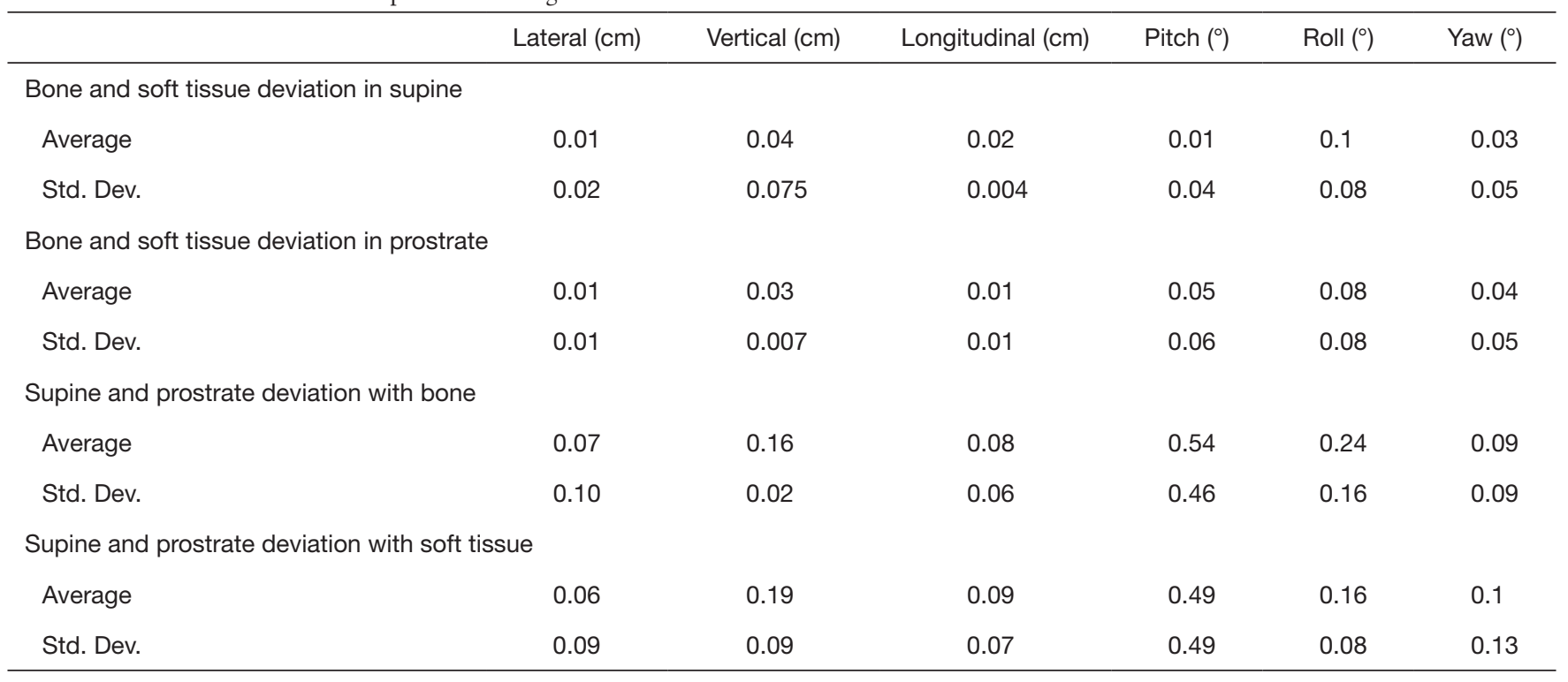

Table 3 Statistics results from 100 supine and 100 prostrate matching data of 14 real patients

\begin{tabular}{lccccc}
\hline & Lateral $(\mathrm{cm})$ & Vertical $(\mathrm{cm})$ & Longitudinal $(\mathrm{cm})$ & Pitch $\left(^{\circ}\right)$ & Roll $\left(^{\circ}\right)$ \\
\hline Supine & & & & & \\
Yverage & -0.07 & 0.14 & -0.12 & 0.79 & 0.41 \\
Std. Dev. & 0.22 & 0.35 & 0.4 & 1.6 & 0.71 \\
Prostrate & & & & 0.03 \\
Average & -0.16 & 0.19 & -0.05 & 1.1 & 0.65 \\
Std. Dev. & 0.27 & 0.48 & 0.34 & 1.49 & -0.23 \\
\hline
\end{tabular}

translation direction between different positions is not more than $0.19 \mathrm{~mm}$, and the rotation direction is less than $0.54^{\circ}$.

\section{Patient setup error analysis}

All patients were registered with bone registration. The error in the supine position is from 100 patient data points, while the error in the prone position is from the other 100 patient data points. The average value and standard deviations were calculated, which were considered both in the supine and prone positions, as shown in Table 3. Considering the supine position on 100 3D match plan CT-CBCT, the average \pm SD interfraction displacement in the vertical, longitudinal, and lateral directions was $0.14 \pm 0.35,-0.12 \pm 0.4$ and $-0.07 \pm 0.22 \mathrm{~cm}$, respectively. The mean $( \pm$ SD) interfraction rotations were pitch $=0.79^{\circ} \pm 1.6^{\circ}$, roll $=0.41^{\circ} \pm 0.71^{\circ}$, and yaw $=-0.03^{\circ} \pm 0.8^{\circ}$. In view of the prone position, the average \pm SD of vertical, longitudinal, and lateral directions were $0.19 \pm 0.48$, $-0.05 \pm 0.34$, and $-0.16 \pm 0.27 \mathrm{~cm}$, respectively. The means $( \pm \mathrm{SD})$ were pitch $=1.1^{\circ} \pm 1.49^{\circ}$, roll $=0.65^{\circ} \pm 1^{\circ}$, and yaw $=-0.23^{\circ} \pm 0.75^{\circ}$. Then, all the data in the supine position and prone position were analyzed by pairwise correlation analysis. The correlation coefficient $\mathrm{r}$ and $\mathrm{p}$ value can be found in Table 4.

As shown in Table 4, when the patient is in the supine position, there is a significant moderate correlation between the longitudinal and the pitch direction, but a weak correlation was found between the other directions where $\mathrm{P}<0.05$. Similarly, when the patient is in the prone position, only the vertical and pitch direction has a significant moderate correlation. The other directions have a weak correlation. 
Table 4 The $\mathrm{r}$ and $\mathrm{P}$ value between the six directions of the pelvic patients

\begin{tabular}{|c|c|c|c|c|}
\hline & \multicolumn{2}{|c|}{ Supine } & \multicolumn{2}{|c|}{ Prostrate } \\
\hline Vertical-Longitudinal & 0.695 & 0.040 & 0.517 & -0.066 \\
\hline Vertical-Lateral & 0.004 & 0.288 & 0.099 & -0.166 \\
\hline Vertical-Pitch & 0.121 & 0.156 & 0 & -0.441 \\
\hline Vertical-Yaw & 0.208 & -0.127 & 0 & 0.348 \\
\hline Longitudinal-Lateral & 0.245 & 0.117 & 0.293 & -0.106 \\
\hline Longitudinal-Pitch & 0 & 0.446 & 0.501 & 0.068 \\
\hline Longitudinal-Roll & 0.045 & -0.201 & 0.757 & 0.031 \\
\hline Lateral-Roll & 0 & -0.383 & 0.476 & 0.072 \\
\hline Lateral-Yaw & 0.020 & -0.233 & 0.008 & -0.263 \\
\hline Pitch-Roll & 0.001 & -0.329 & 0.734 & 0.034 \\
\hline Pitch-Yaw & 0.134 & -0.151 & 0.276 & -0.110 \\
\hline Roll-Yaw & 0.867 & -0.017 & 0.07 & -0.268 \\
\hline
\end{tabular}

\section{Discussion}

In this work, the known errors introduced in the phantom experiment were detected after registration between CBCT and planning CT. After setup error correction, the 6-DoF couch based on CBCT exhibited high accuracy. We also find that the average error shift caused by the different registration methods is less than $0.04 \mathrm{~cm}$ in the translation direction and $0.1^{\circ}$ in the rotational direction when phantom positions were kept unchanged. This result indicated that the registration method is a factor of random error for pelvic patients, which requires that the same registration method be selected when CBCT registration is performed in clinical practice. Schmidhalter et al. also reported that the use of matching methods is one of the error sources (1). On the other hand, the average deviation caused by different positions does not exceed $0.19 \mathrm{~cm}$ in the translation direction, and the rotation direction is less than $0.54^{\circ}$ under the same registration method. Different postures may lead to different degrees of error in all directions. It is impossible to determine which posture error is smaller than the others. To some extent, posture fixation may be determined according to the patient's situation.

Interestingly, the error of the rotation direction remains almost unchanged when the deviation of the translation direction (lateral, vertical, longitudinal) is introduced in the supine position. This shows that the translation direction does not lead to an error in the rotation direction. However, when an angle of $2^{\circ}$ in pitch is introduced, the error increases in the longitudinal and vertical directions. When the pitch and yaw directions are introduced at the same time, the errors of the three translation directions increase, and the rolling direction errors remain unchanged. When the lateral direction, pitch direction and yaw direction are all introduced at the same time, the increasing trend of other direction errors is consistent with the error change trend caused by introducing pitch and yaw direction errors. This indicates that these errors are caused by the rotation direction. The phenomena described above also exist in the prone position. It should be noted that there may be some relationship between the errors in all six directions.

To confirm the phenomena observed in the phantom experiment, we retrospectively analyzed the data of 200 groups with different postures in 14 cases. The average value and standard deviations were calculated both in the supine and prone positions. Additionally, a correlation analysis between six directions was performed. The 
average value and standard deviations of supine patients in translation (vertical, longitudinal, and lateral) and rotation (pitch, roll and yaw) directions were $0.14 \pm 0.35,-0.12 \pm 0.4$, $-0.07 \pm 0.22 \mathrm{~cm}, 0.79^{\circ} \pm 1.6^{\circ}, 0.41^{\circ} \pm 0.71^{\circ}$, and $-0.03^{\circ} \pm 0.8^{\circ}$, respectively. For the prone position, the average \pm SD of the vertical, longitudinal, and lateral directions were $0.19 \pm 0.48$, $-0.05 \pm 0.34$, and $-0.16 \pm 0.27 \mathrm{~cm}$, respectively. The mean $( \pm$ SD) of pitch, roll and yaw was $1.1^{\circ} \pm 1.49^{\circ}, 0.65^{\circ} \pm 1^{\circ}$, and $-0.23^{\circ} \pm 0.75^{\circ}$, respectively. This result indicated that the errors in the prone position (except longitudinal) are larger than those in the supine position. One possible reason is that the fixed supine position is more likely to lead to random error in the longitudinal axes (2).

Few studies of the correlation between the errors of the six dimensions of the 6-DoF couch have been reported, especially for pelvic tumors. Chiesa et al. reported that there is an extremely weak correlation between translational and rotational errors in prostate treatment (all $\mathrm{R}$ values $<0.2)(2)$. However, we found that there is a significant moderate correlation between the longitudinal and pitch directions and between the vertical and pitch directions when the patient is in the supine position. This may be caused by the limited existing data of rotational errors or correlation between translational and rotational shift.

According to Stroom et al. (16) and van Herk et al.'s (17) research on patient positioning error, the average of all the positioning errors indicates the systematic error $(\Sigma)$, and the random error is represented by the standard deviation of all the positioning errors $(\sigma)$. Although some margin formulas were proposed (18), the margins of PTV are often calculated as follows: $M_{p t v}=2.5+0.7 \sigma$. Then, the $M_{p t v}$ values of the $\mathrm{x}^{-}, \mathrm{y}-$, and $\mathrm{z}$-axes are calculated. However, we find that there is a certain relationship between the 6-DoF errors of the six-dimensional couch, and the error of the rotation direction during the positioning process may cause the change of the target position even within the tolerance range. Therefore, when calculating the PTV margins, one must consider not only the respective errors in each direction but also the influence of different directions. This assumption should be confirmed in future studies.

\section{Conclusions}

By using phantom experiments and patient studies, we show that the six-dimensional couch positioning verification system based on CBCT has high accuracy and can meet the requirements of precise radiotherapy for pelvic tumors. It is suggested that the six-dimensional couch based on
CBCT should be used for pre-radiotherapy positioning in the clinic. We found that there is a significant moderate correlation between the longitudinal and pitch directions when the patient is in the supine position and the vertical and pitch directions when the patient is in the supine position. It is indicated that no matter what position is selected for fixation, there is always a certain relationship between the translation direction and the rotation direction.

\section{Acknowledgments}

Funding: This research was partly supported by the National Nature Science Foundation of China (Grant No. 81901743), the WBE Liver Fibrosis Foundation, China (Grant No. CFHPC2019027) and the Key Research and Development Program of Shandong Province (Grant No. 2019GSF108134).

\section{Footnote}

Data Sharing Statement: Available at http://dx.doi. org/10.21037/tcr-20-1528

Conflicts of Interest: All authors have completed the ICMJE uniform disclosure form (available at http://dx.doi. org/10.21037/tcr-20-1528). The authors have no conflicts of interest to declare.

Etbical Statement: The authors are accountable for all aspects of the work in ensuring that questions related to the accuracy or integrity of any part of the work are appropriately investigated and resolved. The study was conducted in accordance with the Declaration of Helsinki (as revised in 2013). The ethics committee of Shandong Cancer Hospital and Institute approved this study (No.: 2019-371). Because this is not a treatment-based study, our institutional review board waived the need for written informed consent from the participants. However, the patient information was kept anonymous to protect their confidentiality.

Open Access Statement: This is an Open Access article distributed in accordance with the Creative Commons Attribution-NonCommercial-NoDerivs 4.0 International License (CC BY-NC-ND 4.0), which permits the noncommercial replication and distribution of the article with the strict proviso that no changes or edits are made and the original work is properly cited (including links to both the formal publication through the relevant DOI and the license). 
See: https://creativecommons.org/licenses/by-nc-nd/4.0/.

\section{References}

1. Schmidhalter D, Fix MK, Wyss M, et al. Evaluation of a new six degrees of freedom couch for radiation therapy. Med Phys 2013;40:111710.

2. Chiesa S, Placidi L, Azario L, et al. Adaptive optimization by 6 DOF robotic couch in prostate volumetric IMRT treatment: rototranslational shift and dosimetric consequences. J Appl Clin Med Phys 2015;16:35-45.

3. Schmidhalter D, Malthaner M, Born EJ, et al. Assessment of patient setup errors in IGRT in combination with a six degrees of freedom couch. Z Med Phys 2014;24:112-22.

4. Mancosu P, Reggiori G, Gaudino A, et al. Are pitch and roll compensations for patient repositioning: a data analysis with CBCT over 2945 fractions from different locations. Brit J Radiol 2015;88:1-14.

5. Finnigan R, Lamprecht B, Barry T, et al. Inter-and intrafraction motion in stereotactic body radiotherapy for spinal and paraspinal tumours using cone-beam CT and positional correction in six degrees of freedom. J Med Imaging Radiat Oncol 2016;60:112-8.

6. Cheon W, Cho J, Ahn SH, et al. High-precision quality assurance of robotic couches with six degrees of freedom. Phys Med 2018;49:28-33.

7. Guckenberger M, Meyer J, Wilbert J, et al. Precision of image-guided radiotherapy (IGRT) in six degrees of freedom and limitations in clinical practice. Strahlenther Onkol 2007;183:307-13.

8. Spadea MF, Baroni G, Riboldi M, et al. Benefits of six degrees of freedom for optically driven patient set-up correction in SBRT. Technol Cancer Res Treat 2008;7:187-95.

9. Yao L, Zhu L, Wang J, et al. Positioning accuracy during VMAT of gynecologic malignancies and the resulting dosimetric impact by a 6-degree-of-freedom couch in

Cite this article as: Fu C, Ma C, Shang D, Qiu Q, Meng H, Duan J, Yin Y. Geometric accuracy evaluation of a sixdegree-of-freedom (6-DoF) couch with cone beam computed tomography (CBCT) using a phantom and correlation study of the position errors in pelvic tumor radiotherapy. Transl Cancer Res 2020;9(10):6005-6012. doi: 10.21037/tcr-20-1528 combination with daily kilovoltage cone beam computed tomography. Radiat Oncol 2015;10:104.

10. Zhang Q, Driewer J, Wang S, et al. Accuracy evaluation of a six-degree-of-freedom couch using cone beam CT and IsoCal phantom with an in-house algorithm. Med Phys 2017;44:3888-98.

11. Dhabaan A, Schreibmann E, Siddiqi A, et al. Six degrees of freedom CBCT-based positioning for intracranial targets treated with frameless stereotactic radiosurgery. J Appl Clin Med Phys 2012;13:3916.

12. Hyde D, Lochray F, Korol R, et al. Spine stereotactic body radiotherapy utilizing cone-beam CT image-guidance with a robotic couch: intrafraction motion analysis accounting for all six degrees of freedom. Int J Radiat Oncol Biol Phys 2012;82:e555-62.

13. Stieb S, Malla M, Graydon S, et al. Dosimetric influence of pitch in patient positioning for radiotherapy of long treatment volumes; the usefulness of six degree of freedom couch. Br J Radiol 2018;91:20170704.

14. Talbot J, Meyer1J, Watts R, et al. A method for patient set-up guidance in radiotherapy using augmented reality. Australas Phys Eng Sci Med 2009;32:203-11.

15. Archer BR, Glaze S, North LB, et al. Dosimeter placement in the Rando phantom. Med Phys 1977;4:315-8.

16. Stroom JC, Heijmen BJM. Geometrical uncertainties, radiotherapy planning margins, and the ICRU-62 report. Radiother Oncol 2002;64:75-83.

17. van Herk M. Errors and margins in radiotherapy. Semin Radiat Oncol 2004;14:52-64.

18. Naoi Y, Kunishima N, Yamamoto K, et al. A planning target volume margin formula for hypofractionated intracranial stereotactic radiotherapy under cone beam CT image guidance with a six-degrees-of-freedom robotic couch and a mouthpiece-assisted mask system: a preliminary study. Br J Radiol 2014;87:20140240. 